\title{
MAIS TONELADAS POR MEGAWATT: COMO PRODUZIR MAIS CONSUMINDO MENOS ENERGIA, COM O AUXÍLIO DE SISTEMAS DE AUTOMAÇÃO E DE GESTÃO DA PRODUÇÃO*
}

\author{
Bruno Aguiar do Prado ${ }^{1}$ \\ Fernando José Ferrarezzi \\ Renzo Bottino Ziegler ${ }^{3}$ \\ Wanderson Silveira Alvarenga ${ }^{4}$
}

\begin{abstract}
Resumo
Atividades industriais, especialmente relacionadas ao segmento de MMM, têm força econômica considerável além de significativa relevância no cotidiano da população. Neste contexto a disseminação da importância do uso sustentável dos recursos energéticos vem sendo um grande destaque. Diante da necessidade em se atingir a eficiência energética de forma plena, a indústria tem investido em infraestrutura e equipamentos que tem o objetivo de disponibilizar as informações de consumos e demandas de equipamentos e linhas de produção através de medidores inteligentes e conectividade entre as "ilhas" de informação espalhadas no site. A fim de relacionar e disponibilizar os dados obtidos e transformá-los em informação existem diversos softwares com a capacidade de gerar e correlacionar KPIs e análises, evidenciando gaps, direcionando melhorias e até mesmo dar suporte à priorização de investimentos. Esse tipo de abordagem é de extrema importância para que se possam alcançar economias significativas para todas as operações do segmento de MMM.
\end{abstract}

Palavras-chave: MES; Mineração; Eficiência energética.

\section{MORE TONS PER MEGAWATT: HOW TO PRODUCE MORE CONSUMING LESS ENERGY, USING AUTOMATION AND PRODUCTION MANAGEMENT SYSTEMS Abstract}

Industrial activities, especially those related to MMM segment, have considerable economic strength as well as significant relevance in daily life of the population. In this context the spread of the importance of sustainable use of energy resources has a major highlight. Faced with the need to fully achieve energetic efficiency, the industry has been investing in infrastructure and equipments that provide information concerning consumption and demand about equipment and process lines through smart meters and connectivity between "islands" of information scattered throughout the site. In order to relate data from plant and transform them into information, there are a great variety of software applications that have the ability to generate and correlate KPIs and analysis that has capacity to show gaps, drive improvements and even support the prioritization of investments. This approach is extremely important so that we can achieve very significant savings for all MMM segment operations.

Keywords: MES; Mining; Energy efficiency.

Engenheiro Controle e Automação. Engenheiro de Aplicação. Schneider-Electric, Belo Horizonte, $M G$, Brasil.

Engenheiro Eletricista. Engenheiro de Aplicação. Schneider-Electric, São Paulo,SP, Brasil.

Engenheiro Eletrônico. Coordenador de Serviços MES. Schneider-Electric, São Paulo,SP, Brasil.

Sistemas de Informação. Arquiteto de Soluções, Schneider-Electric, Belo Horizonte, MG, Brasil. 


\section{INTRODUÇÃO}

As atividades envolvidas no segmento de MMM (Metalurgia, Materiais e Mineração) correspondem a 3\% do PIB mundial (2014) - onde a mineração corresponde a 0,8\% deste montante, abrangendo um mercado estimado em 1,9 trilhão de euros (2014). Além da força econômica deste segmento, a importância no cotidiano da população mundial fica evidenciada num estudo da OECD, que declara que um cidadão americano nascido nos dias de hoje irá demandar cerca de 1,36Mt de minerais, metais e combustíveis ao longo de sua vida [1]. Se levarmos em conta que a população mundial continua a crescer, sendo estimado um crescimento de $12 \%$ de seu número absoluto até 2025 , além do aumento da urbanização e do número de habitantes considerados sendo da classe média (50\% da população mundial em 2025), fica clara a importância e a força do segmento de MMM na economia mundial.

No Brasil, a situação não é diferente. A participação da mineração no PIB nacional é ainda maior, chegando a 4,4\%, além de $22,4 \%$ das exportações nacionais (2010). 0 minério de ferro corresponde a $60 \%$ da produção mineral brasileira, e ainda gera cerca de 29,2 mil empregos diretos e indiretos, destacando sua importância na economia brasileira [2].

A disseminação da importância do uso sustentável dos recursos energéticos está em grande destaque atualmente, em função das dificuldades de sustentabilidade da matriz energética brasileira face às vissicitudes climáticas ao longo dos últimos anos, porém não teve o mesmo realce dentro da indústria nacional nos últimos anos, mesmo sendo este setor o maior consumidor de energia no país, respondendo por $40,7 \%$ de seu total (2008). Os setores residencial, comercial e público recebem maior prioridade nas políticas governamentais de eficiência energética, apesar de responderem por apenas $15,8 \%$ do total de consumo de energia no Brasil. E ainda, é o setor industrial o que apresenta o maior potencial de economia de energia. De acordo com um estudo da PROCEL Indústria de 2009, existe um potencial técnico de redução de 25,7\% (ou 14,6 Mtep - tonelada equivalente de petróleo) do consumo global de energia na indústria brasileira [3].

No segmento de MMM, um grande percentual dos custos de produção advem do custo de energia. Porém, os benefícios que a eficiência energética poderia trazer nunca foram atingidos, devido a um histórico de preços baixos de energia, a um foco na rápida expansão da produção guiada pelas demandas do mercado, e a uma escala pequena e discreta da maioria dos projetos de eficiência. Além disso, várias barreiras foram levantadas no mesmo estudo da PROCEL Indústria de 2009, com destaque para [4]:

Mineração:

- Necessidade de maior difusão das tecnologias mais eficientes e dos potenciais benefícios;

- Percepção de riscos altos com a introdução de novas tecnologias;

- Baixa liquidez e restrições de acesso a linhas de financiamento;

- Necessidade de treinamento de pessoal para identificar oportunidades de eficiência energética e para fazer a gestão dos projetos que se mostrarem viáveis.

Siderurgia:

- Racionalização do uso de energia compete com outras prioridades de investimento. Dificuldade para viabilizar soluções de alto custo; 
- Percepção de alto risco tecnológico para substituição dos equipamentos de grande porte utilizados no setor;

- Acesso restrito a informações por empresas de menor porte;

- Excessiva burocracia para liberação de recursos de financiamento;

- Prioridade para a redução de riscos na ampliação de instalações de cogeração.

Metais não-ferrosos, ferro-ligas e fundição:

- Estrutura legal pouco atrativa para cogeração ou produção de energia independente;

- Necessidade de treinamento de pessoal para identificar oportunidades de eficiência energética e para fazer a gestão dos projetos que se mostrarem viáveis;

- Indisponibilidade de determinadas tecnologias;

- Racionalização do uso de energia compete com outras prioridades de investimento;

- Elevados investimentos iniciais;

- Incertezas quanto aos preços da energia e altos custos iniciais dos investimentos devido aos impostos de importação;

- Restrições ao financiamento (específico para o setor de ferro-ligas).

Com a mudança deste cenário atualmente, com a desaceleração do crescimento econômico mundial, crise econômica interna e aumento dos preços da energia (tanto elétrica quanto térmica), a eficiência energética ganha destaque, gerando ainda outros possíveis benefícios, como aumento da produtividade e redução de emissões de CO2. E é possível obter grandes reduções de consumo energético com investimentos de pequenos montantes de capital.

Nosso objetivo neste trabalho é apresentar o atual cenário de medição e controle do consumo energético, e como sistemas de automação e sistemas de execução da manufatura (MES, da sigla em inglês) podem facilitar a visualização dos dados de consumo e levar à tomada de decisões que levem a uma maior eficiência energética.

\section{MATERIAIS E MÉTODOS}

A medição do uso da energia tem evoluído muito ao longo do tempo e atualmente já existem inúmeros medidores digitais, para as mais diversas classes de aplicação, e com uma grande variedade de possibilidades de comunicação (Ethernet, ModBus, etc.) além da possibilidade do uso de telemetria para aquisições à distância e sem a necessidade de uma infraestrutura no local.

Alguns medidores evoluíram muito em relação aos seus antepassados medidores analógicos, e se tornaram grandes centros de informações instantâneas não somente do consumo energético realizado, mas também a qualidade desse consumo, com medições muito precisas de fatores de potência e carga consumidas. Existe uma tríplice preocupação correlacionada à medição do uso de energia, onde temos os seguintes pilares: 


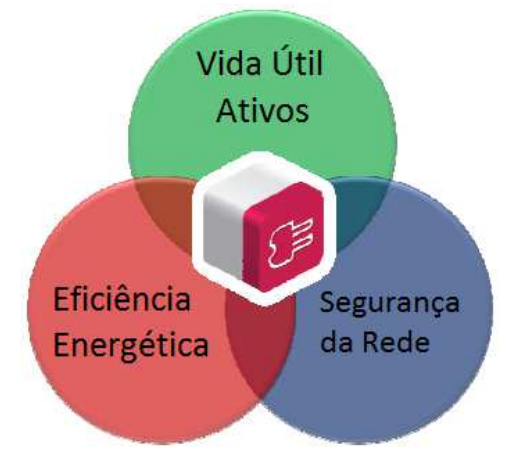

Figura 1. Pontos Críticos no Gerenciamento de Energia

- Vida Útil dos Ativos:

- Análise de alimentação e capacidade do circuito;

- Monitoramento de baterias, motores, UPS, geradores;

- Estudos de balanço de fase;

- Análise de harmônicas;

- Manutenção preditiva.

- Eficiência Energética

- Estimativa de contas;

- Alocação de custos;

- Relatórios WAGES

- Gerenciamento da demanda;

- Relatórios de fator de potência.

- Segurança da Rede

- Monitoramento e relatórios de distribuição elétrica;

- Alarmes antecipados;

- Analise pós-evento;

- Relatórios de evento;

- Monitoramento e relatórios da qualidade da potência contratada.

ॠSigla para itens de energia industriais: Water (água), compressed $\underline{A}$ ir (ar comprimido), Gas (gás), Eletricity (eletricidade) e $\underline{\text { Steam (vapor) }}$

Contudo a instalação desses medidores ainda segue a conveniência orientada principalmente à vida útil dos equipamentos e à existência de uma rede segura e confiável. Dessa forma os medidores são principalmente ligados aos centros elétricos, como substações, transformadores, galpões e CCMs. Em alguns casos, somente equipamentos de altíssima demanda possuem medidores próprios.

Essa estratégia de medição na maioria das situações pode impossibilitar uma série de análises mais efetivas e assertivas do ponto de vista da eficiência energética atrelada à produtividade do equipamento ou site.

Outras fontes energéticas, que não são puramente elétricas, também tem foco maior na segurança das instalações e consumos gerais do que a sua correlação com a produtividade e consumos específicos. Isso ocorre principalmente por existirem verdadeiras "ilhas" de informação na planta, sem que haja sistemas unificados de medição instantânea que possam correlacionar os dados disponíveis nas plantas e apresentá-los em uma forma intuitiva, dando suporte à tomada de decisão.

O Brasil possui diversos exemplos de projetos de eficiência energética industrial, a maioria deles desenvolvido dentro das regras do Programa de Eficiência Energética - PEE (Lei 9.991/00) [2]. Num universo de 217 projetos analisados, o valor investido foi de $R \$ 161$ milhões, gerando uma economia de 626GWh, ou seja, $R \$ 79,00 / \mathrm{MWh}$. 
Grande parte dos projetos caminhou para a economia de eletricidade, próximo de $50 \%$, e apenas uma pequena parte focou na otimização de processos térmicos (6\%). Nestes projetos, uma situação típica ocorreu em uma empresa do ramo de Metais que necessitava aprimorar o gerenciamento da energia elétrica utilizada pela companhia onde, por várias vezes, a produção era reduzida para não ultrapassar as cargas contratadas.

Após uma análise criteriosa, foi constatado que o consumo de energia do site era medido na entrada dos galpões. Medidores unitários existiam somente em poucos equipamentos que, historicamente, eram os maiores consumidores de energia elétrica. Ainda assim, parte das informações era registrada manualmente e a outra parte era historiada em bancos de dados da equipe de manutenção. Esses valores eram depois rateados por departamento, basicamente atrelados a testes históricos de consumo. Não havia então nenhuma correlação entre a produção e o consumo de energia elétrica.

Mesmo sem o investimento para inclusão de mais medidores, foi utilizado um sistema MES para agregar as informações para cada lote produzido:

- Tempo do ciclo de produção por cada etapa;

- Consumo de energia por lote produzido;

- Total produzido;

- Qualidade do material produzido;

- Todos os valores obtidos podendo ser discretizados por TURNO e TURMA. A apresentação destas informações de forma correlacionada permitiu a inclusão de metas de consumo de energia por tipo de produto produzido, o que fez com que a equipe de operação/manutenção sempre buscasse tais metas.

O fruto dessa iniciativa foi a padronização na forma de produção, já que diferentes operadores possuíam diferentes formas de trabalho, que, por não terem conhecimento, acarretavam em maiores gastos de energia.

Um outro projeto que serve de exemplo diz respeito ao consumo de gás natural na etapa de laminação de uma empresa de Metalurgia. No cenário inicial investigado o consumo de gás natural em uma etapa de aquecimento das peças (responsável por consumos acima de $50 \%$ desse insumo) aparentemente possuía um cenário favorável para análise de produtividade atrelada ao consumo desse item, já que cada um dos fornos possuia um medidor individual de consumo de gás.

Contudo, pela estratégia do processo, cada lote que passava pelo forno só possuía o tempo que essa peça passou pelo equipamento. $E$ isso ocorria somente pela primeira passagem, já que depois de laminar a peça poderia, na maioria das vezes, ter que retornar para esses fornos para um reaquecimento.

Dessa forma, ainda que fosse possível a análise correta do consumo de gás atrelado a cada tonelada produzida pelo equipamento, os lotes recebiam valores "rateados" de consumo de gás, atrelados basicamente ao tempo total entre o lote entrar no forno e sair pela última vez no laminador, e o consumo histórico de gás medido nessa área da planta.

Esse é um caso em que a medição é feita corretamente, mas que a correlação de dados não é feita de forma adequada, prejudicando, por exemplo, a análise precisa do custo de produção de cada produto ofertado no mercado.

Diante dos cenários descritos acima e de muitos outros relacionados à ineficiência energética, temos como modelo para a busca a excelência neste quesito, a fim de alcançar os melhores resultados, o foco em quatro passos [5]: 


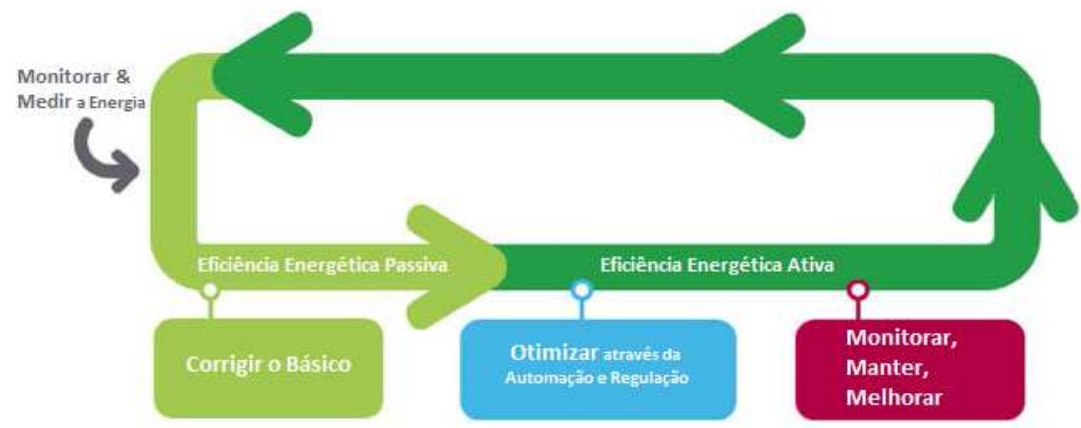

Figura 2. Sistema de Quatro Passos

- Passo 1 - Monitorar e Medir: Identificação dos pontos chave de medição e métodos a serem utilizados (medidores instantâneos, coletas periódicas);

- Passo 2 - Corrigir o Básico: Correções pontuais e mais simplificadas, como vazamentos, redução de harmônicas e correção de fator de potência;

- Passo 3 - Automatizar e Otimizar: Utilização de automação básica para controlar processos simples de produção (em especial tempo e momentos que equipamentos devem ser - ou não - ligados) e processos avançados de automação que utilizam diversos modelos de otimização de sistemas para também chegar nos pontos ótimos de utilização dos ativos;

- Passo 4 - Monitorar, Manter, Melhorar: Para prover a melhoria contínua é feito o uso da inteligência montada para gerenciamento de energia com sistemas de gerenciamento da produção, a fim de prover informações contextualizadas do uso da energia, promovendo constante monitoramento e identificação de oportunidades de melhorias, além de ferramentas para suporte à tomada de decisão.

\section{RESULTADOS E DISCUSSÃO}

Uma empresa que atinge a maturidade no gerenciamento energético pode ter benefícios consideráveis ao combinar suas ações com o uso de um sistema de gerenciamento da produção (MES).

Com o uso de modernos sistemas MES, através de uma interface web ou via aplicação desktop, é possível obter dados confiáveis e atualizados de:

- Medições de produtividade com indicação das variações em relação às metas;

- Medições de tempos de paradas, com suas classificações para posterior estratificação;

- Medições de consumo de energia com relação a metas e previsões de demanda;

- Medições do consumo de insumos relacionado aos picos e demanda de energia dos processos. 


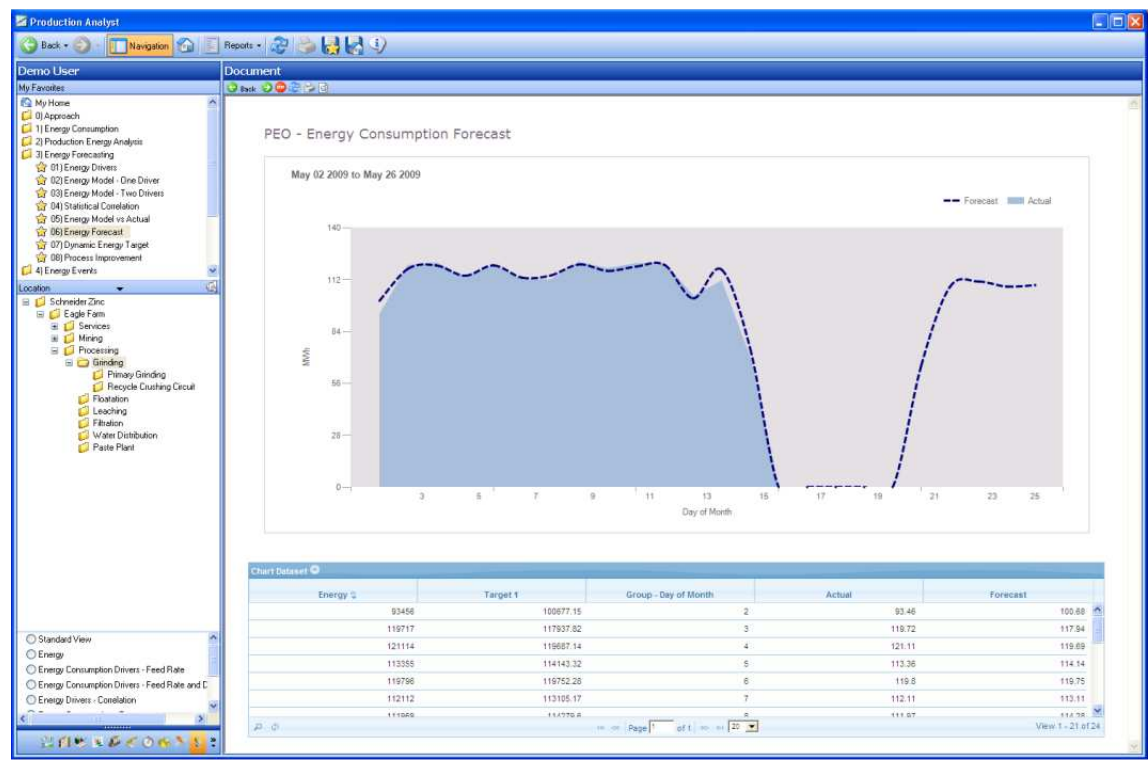

Figura 3. - Exemplo de Tela de Relação de previsão de consumo pelo realizado [6].

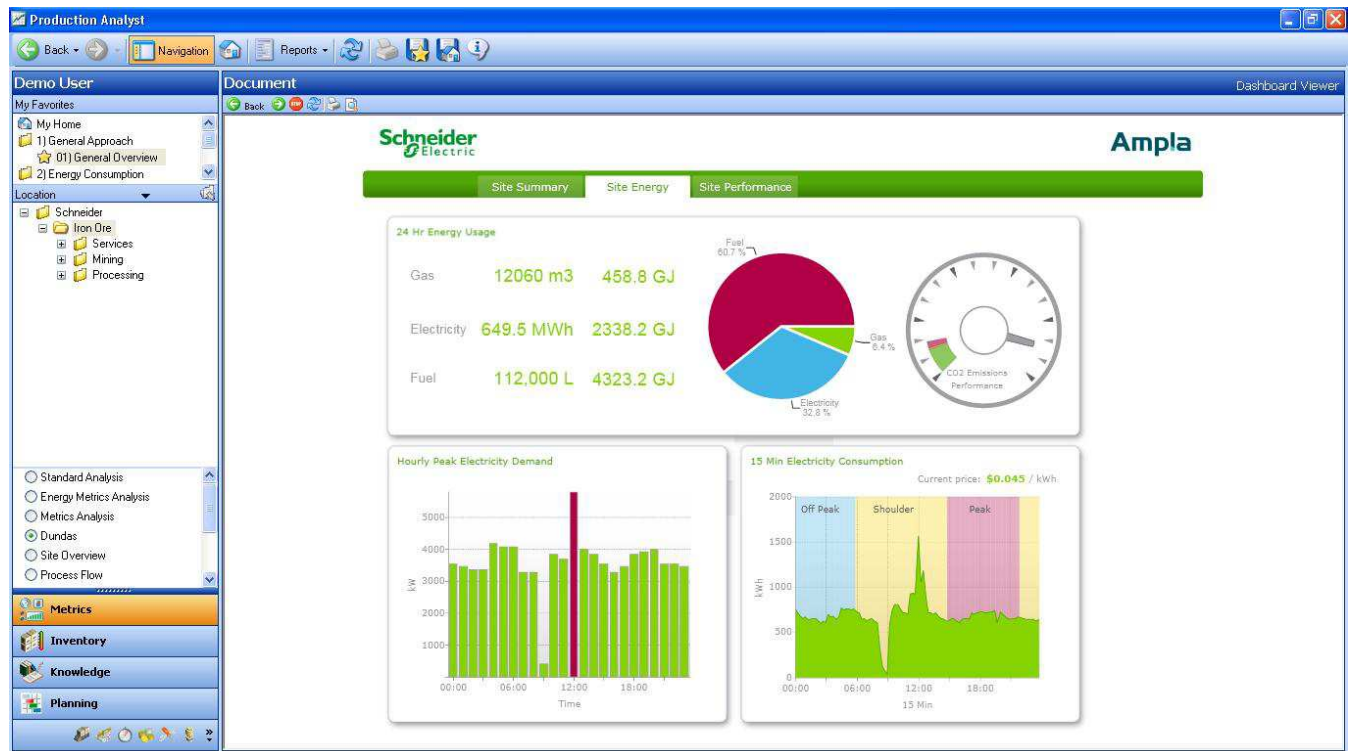

Figura 4. - Exemplo de dashboard relacionando o consumo de insumos com os picos e demandas de energia [6].

Uma vez que dados de produção (proveniente de balanças, medidores de vazão, horímetros e etc) e dados de consumo de energia (provenientes de medidores de energia dispostos no site) estejam disponíveis em uma base de dados, o sistema MES é capaz de prover facilmente análises contextualizadas, transformando os dados obtidos em informação indispensável para a tomada e decisão. Comumente na indústria, a base de dados é fornecida através de um sistema supervisório ou de um sistema historiador (PIMS, da sigla em inglês) que disponibiliza estes dados através do protocolo OPC (OLE for Process Control), protocolo aberto e difundido pelo mercado de automação industrial para troca de informações em tempo real. $O$ licenciamento da ferramenta é realizado através de pontos de medição, que podem ser distribuídos dentro dos módulos (Energia, Produção, Qualidade, Métricas, Paradas, Planejamento, etc.) existentes, e licenciamento de acesso simultâneo pelos clientes.

Abaixo segue esquema da arquitetura de Hardware tipicamente envolvida em um projeto desse tipo: 


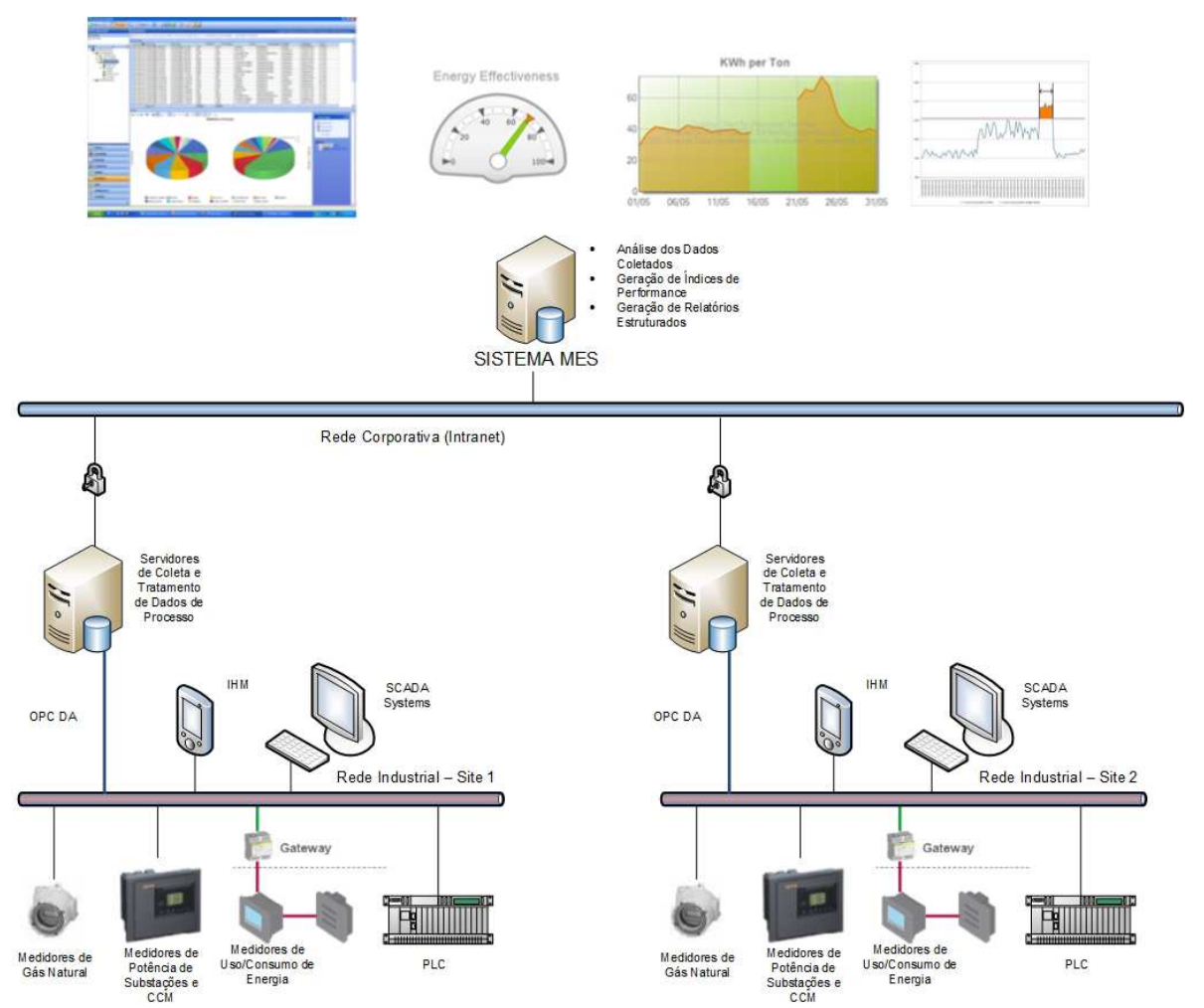

Figura 5. Exemplo de Arquitetura de Hardware

As informações geradas pelo sistema MES também são fonte de informação para sistemas ERP (Enterprise Resource Planning) tais como o SAP e similares. Neste contexto a integração entre ambos é desenhada da forma mais otimizada possível, de modo a atender às necessidades (como por exemplo, apontamentos de consumo energético) e garantir performance, seja ela via banco de dados (OLE DB) ou através de Web Services (XML).

As informações geradas são comumente chamadas de KPI (Key Performance Indicator). Neste contexto, abaixo temos alguns exemplos de KPIs:

- Total de energia consumida por unidade de produção: Esse item pode ser gerado através de qualquer medidor de energia que se faça interesse, resultando em valores de kWh/tonelada, GJ/tonelada e/ou m3/tonelada;

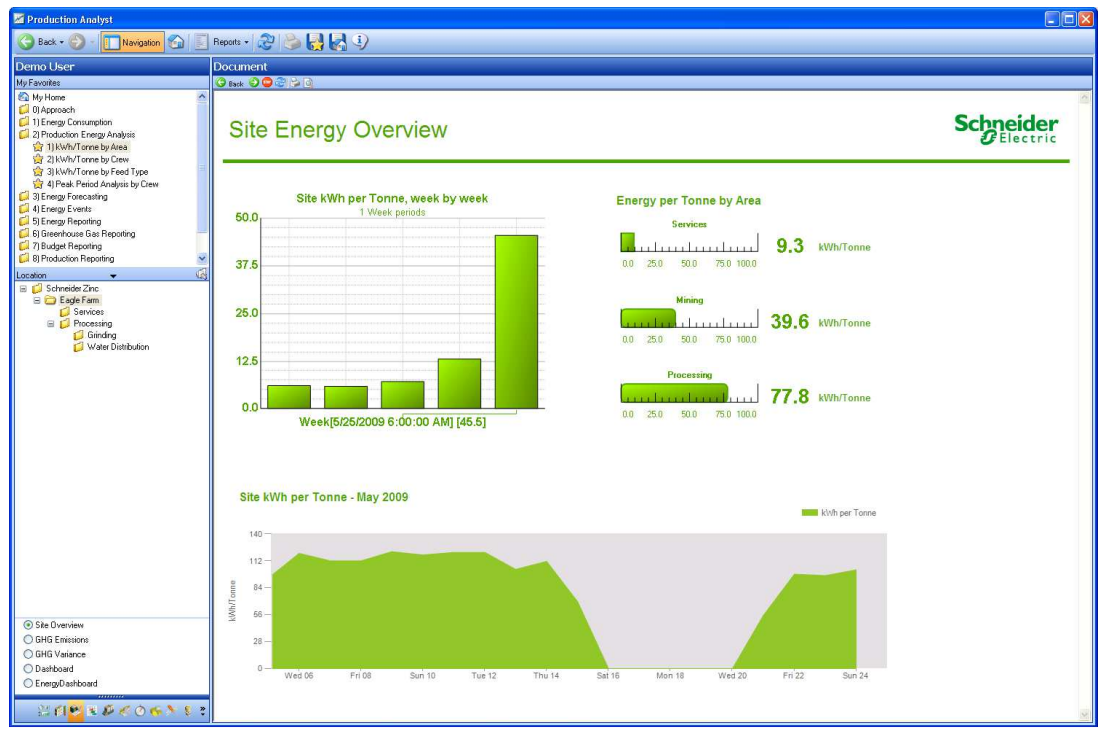

Figura 6. Exemplo de KPI de Consumo de Energia por Tonelada [6]. 
Essa análise pode ser mais bem detalhada quando for separado por unidades de interesse, como Turno ou matéria-prima.

- Consumo de energia durante paradas de equipamentos: Uma vez que todas as paradas estão sendo registradas, é importante realizar um cruzamento do total de energia gasta durante eventos de paradas, gerando índices estratificados por tipo de evento de parada;

- Consumo Excessivo de Energia: De posse dos dados de produção e taxas meta de consumo de energia esse índice indicará o total de energia consumida acima do esperado para um volume de produção. Esse índice baseia-se nas anotações automáticas do sistema MES:

- Data/Hora de Início do sobreconsumo;

- Data/Hora de Fim do sobreconsumo;

- Duração do Evento;

- Quantidade do sobreconsumo.

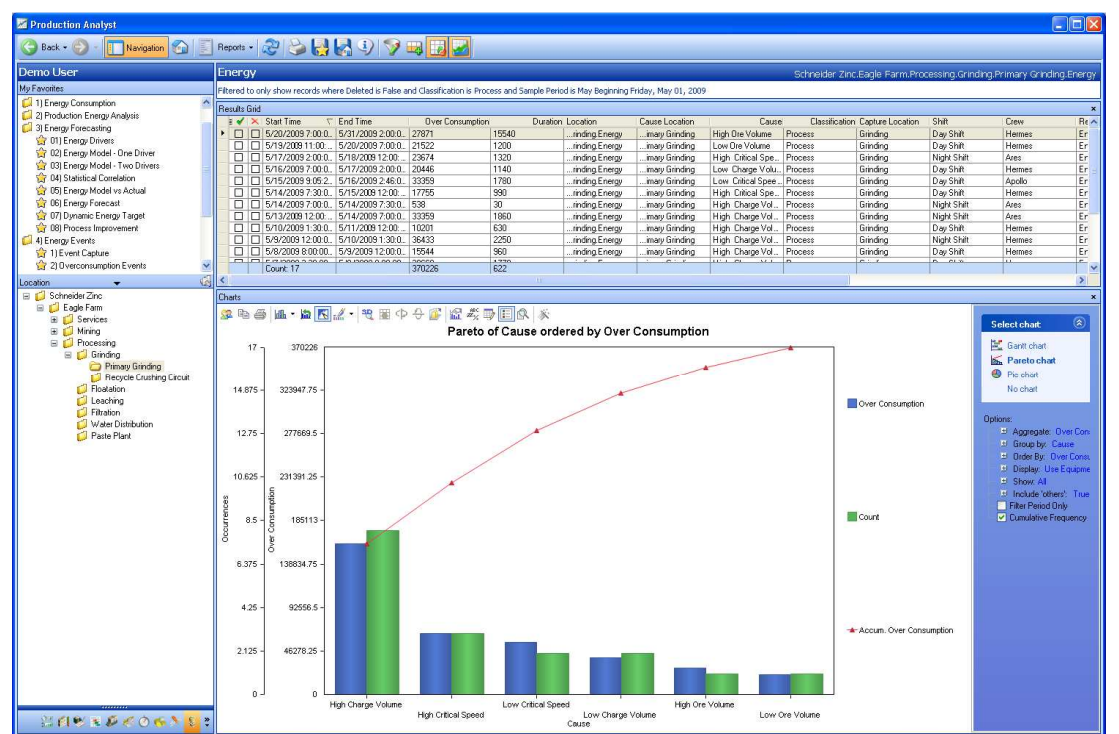

Figura 7. Exemplo de Gráfico de Pareto na Classificação do Consumo Excessivo de Energia [6].

O Brasil é um dos centros de excelência global para soluções industriais utilizando tal ferramenta, com equipe altamente capacitada ao qual já realizaram diversas implementações utilizando a solução. Nos projetos executados são realizados treinamentos operacionais e de manutenção da solução, fornecendo ao cliente não só conhecimento de utilização operacional da ferramenta, mas também conhecimento de configuração e administração. Os treinamentos podem ser realizados no site do cliente ou no escritório da fornecedora da solução.

\section{CONCLUSÃo}

A indústria global de MMM iniciou seu enfoque na eficiência energética como uma forma de melhorar a performance geral da companhia. Estratégias de eficiência energética ocorrem ao longo do tempo e geralmente começam com tarefas básicas, como a medição e progressivamente se expandem incluindo ferramentas avançadas de otimização e técnicas atreladas à melhoria contínua das plantas.

Usando modernos sistemas de medição, comunicação e aquisição de dados, uma grande quantidade de informação está agora disponível para análise. Análises usando uma variedade de softwares e ferramentas que provem informações em 
tempo real onde a energia está sendo consumida e sob quais condições. Nesse sentido, as Oportunidades de Eficiência Energética (EEO da sigla em Inglês) são identificadas, melhorias nas plantas são realizadas e o progresso é monitorado ao longo do tempo.

Economias potenciais para qualquer planta irão depender de muitos fatores, mas o método apresentado nesse trabalho pode auxiliar em alcançar economias muito significativas para todas as operações de mineração. Essas economias e vantagens vem em várias formas e são claramente identificadas para uma companhia MMM de sucesso.

\section{REFERÊNCIAS}

1 OECD (2014). Shifting Gear: Policy Challenges for the next 50 Years. OECD Economics Department Policy Notes, No. 24. 2014.

2 PROCEL Indústria. Eficiência energética na indústria: o que foi feito no Brasil, oportunidades de redução de custos e experiência internacional. 2009.

3 Lorentz SV. Avaliação técnico-econômica de projetos de eficiência energética em mineração, utilizando a teoria das opções reais. Dissertação de Mestrado - Escola de Engenharia, Universidade Federal de Minas Gerais. 2012.

$4 \quad$ ICMM. O setor de mineração no Brasil: fortalecimento institucional para o desenvolvimento sustentável. 2013.

5 Mieli F, Bongiovanni M. Production energy optimization in mining. World Mining Congress. 2013.

6 Schneider-Electric. More Megatons per Megawatt. 05/2010 [acesso em 06 Abril. 2015];1:01-12. Disponível em: http://download.schneiderelectric.com/files?p_Reference $=8000 B R 1105 \& p \_E n D o c T y p e=B r o c h u r e \& p \_F i l e \_l d=742$ 11820\&p_File_Name=PEO_8000BR1105.pdf 